\title{
DX PUZZLE: WHERE ARE WE NOW?*
}

\section{Z. WILAMOWSKI}

Institute of Physics, Polish Academy of Sciences

Al. Lotników 32/46, 02-668 Warszawa, Poland

\section{T. SUSKI}

UNIPRESS, Polish Academy of Sciences, Sokołowska 29/37, 01-142 Warszawa, Poland

AND W. JANTSCH

Johannes Kepler University, 4040 Linz, Austria

\begin{abstract}
A brief review of the experimental data on the metastable DX-centers in AlGaAs is presented. The experimental proofs of the two-electron nature and of the intermediate, one-electron state of the DX-centers are discussed. We collect the available experimental data on the ground state, electron-emission and capture energies and we discuss the nature of the lattice barrier. The effect of splitting of these energies in AlGaAs alloys and the consequences of the splitting on the capture and emission kinetics are analyzed. The different character of the barrier and of the alloy splitting for donors of the IV and VI group is underlined. The necessity to consider the interdonor Coulomb interaction when discussing the experimental data is also pointed out.
\end{abstract}

PACS numbers: $72.20 . \mathrm{Jv}, 71.55 .-\mathrm{i}$

\section{Introduction}

The name $D X$-center has been introduced to describe the unusual properties of the center induced by donor doping in AlGaAs [1]. These centers are characterized by, at least, two states: (i) a classical, hydrogen-like, shallow donor, which binds a single electron, and (ii) a deep, DX-state, which is separated by a barrier from the shallow one. In the majority of models used for describing DX properties a large lattice relaxation is postulated, i.e. a local rearrangement of the atoms from the donor vicinity is assumed to be the origin of the barrier, usually called the lattice barrier. The problem of the number of electrons bound on the DX-state has been a subject of discussion for the last few years. Now, it is commonly accepted that two electrons are bound on the DX-state, which means that the energy gain

*This work is supported in part by the Committee for Scientific Research under grants 204839101,201609101 , and by Fonds zur Förderung der Wissenschaftlichen Forschung in Austria. 
resulting from electron-lattice coupling overcomes the energy of Coulomb rcpulsion between the two involved electrons.

The idea of center forming a two-electron (negative IIubbard energy $U$ ) metastable state was introduced by Baraff et al. [2]. This idea was experimentally verified by Watkins, who showed that a vacancy in silicon binds two electrons [3]. The metastability effect was explained by the pseudo-Jahn-Teller effect, i.e. a spontancous breakdown of the local symmetry with the local lattice distortion governed by a minimization of the energy of the two electrons [4]. Some metastable defects were discussed assuming that the local lattice distortion, accompanying the one-electron state in itself, can lead to an effective lattice barrier and strong metastability. Porowski et al. [5], Dmowski et al. [6] and Baj et al. [7] who investigated the metastable defects in III-V and II-VI compounds, later Lang with Logan [8] - for donor centers in III-V - and Piekara et al. [9], Langer et al. [10], and Dmochowski et al. [11] - for donors in ionic $\mathrm{CdF}_{2}$ - tried to explain the metastability in the strong lattice relaxation approach. The coexistence of the shallow and deep states of the same donor center in $\mathrm{CdF}_{2}$ were explained by Langer and Dmochowski [10-12] with the Toyozawa model [13].

The first-principle numerical calculations of the Si-induced DX-center in GaAs were performed by Chadi and Chang [14]. They introduced a model of the two-electron state showing that a large displacement of the cation from the lattice site in [111] direction (the group-IV donor or the neighboring host cation for the group-VI donor) is stabilized by capture of two electrons on the donor center. More precise calculations, which consider not only the two-electron state but also the well-localized one-electron state of the donor, were performed by Dabrowski et al. [15] and fully confirm the microscopic model of Chadi and Chang [14]. Moreover, the configurational diagram (CD) presented by Dabrowski and Scheffler [15] not only explains the two-electron nature of the DX-centers but allows also to analyze the electron capture and emission processes. Although the numerical error is too big to determine the sequence of the one-electron processes resulting in a capture or emission of two electrons with the simultaneous appearance of lattice relaxation, we will try to convince the reader that with the use of this CD the properties of the DX-centers can be classified and the experimental results can be discussed in a comprehensive way.

It appears to be very difficult to present a review of the experimental data for DX-centers. There is a lot of inconsistency in the existing data. We will try to point out the difficulties and complications responsible for this inconsistency:

(i) The variety of experimental techniques which can be applied for the investigation of DX properties is strongly limited. Especially, spectroscopic techniques can be hardly applied. Neither EPR nor well-resolved optical transitions are observed. A broad spectrum of the photoionization cross-section $[16,17]$ and a weak modification of the phonon absorption [18] are the only data which were analyzed. The interpretation of the magnetic circular dichroism spectra is controversial.

(ii) Since the nature of the DX-center is very specific, most of the experimental results should be analyzed with special caution and in a nonstandard way. 
In particular, one should keep in mind the existence of a lattice barrier leading to metastability effects, the two-electron nature of the DX-state, and the alloy splitting of the energy levels. A standard analysis leads easily to misinterpretations and to inaccurate evaluation of the parameters.

(iii) The DX-centers induced by different donors exhibit qualitativcly different properties. Especially, the DX-centers with the group-IV donors differ qualitatively from those with the group-VI donors.

Thus, to solve the DX puzzle, a re-evaluation, reinterpretation and a careful selection of the experimental data is necessary.

In this paper we are going to present and to comment upon the most recent experimental results, taking into account (as much as possible) all the mentioned complications of interpretation. We begin with the presentation of the CD's they form the proper basis for discussion. Next, we analyze the data which allow to specify the details of these diagrams, i.e. the details of the DX nature.

\section{Configurational diagram}

The CD shows the dependence of the total energy of the donor states upon a generalized coordinate describing the local lattice distortion. The energy represents the sum of the electronic energy, the elastic energy of lattice distortion and the energy of electron-lattice interaction. As long as the electron-lattice interaction remains a linear function of distortion, the $\mathrm{CD}$ is composed of shifted parabolas, commonly used e.g. in the analysis of deep impurity states. In the case of large lattice distortion, however, when the lattice distortion becomes of the order of the lattice constant, the elastic energy is no more a quadratic function of distortion and the CD is usually much more complex.

In the case of a two-electron state the diagram is additionally complicated, since one- and two-electron states should be shown separately. Examples of CD for two electrons and one donor center are shown in Fig. 1. The diagram 1a is based on the original diagram calculated by Dabrowski and Schefller [15] for the $\mathrm{Si}$ impurity in $\mathrm{GaAs}$, while the diagram $1 \mathrm{~b}$ representing the properties of the group-VI donor is anticipated basing on the recent experimental data for AlGaAs:Te. Both diagrams are simplified, since only the ground states of each electron configuration are plotted. The shallow donor energy is also omitted as for a large Bohr radius it follows the energy of the conduction band. The solid line describes the energy of the state which binds two electrons, $\mathcal{E}_{D^{-}}$. The minimum occurring at a large distortion corresponds to the DX-state. The dashed line describes the energy of the system with one electron bound on a well-localized $D^{0}$ state, $\mathcal{E}_{D^{0}}$, while the second electron is in the conduction band, $\mathcal{E}_{\mathrm{CB}}$. Dotted lines describe the case when both electrons are in the $\mathrm{CB}$ and they have a negligible kinetic energy. The depicted energy is an algebraic sum of $2 \mathcal{E}_{\mathrm{CB}}$ and the elastic energy of lattice distortion. To account for the kinetic energy of conduction electrons the dashed and dotted lines should be shifted by the kinetic energy of one electron or two electrons, respectively. According to Dabrowski and Scheffler [15] a photoionization of the DX-center corresponds to the transition from the two electron DX minimum to the 


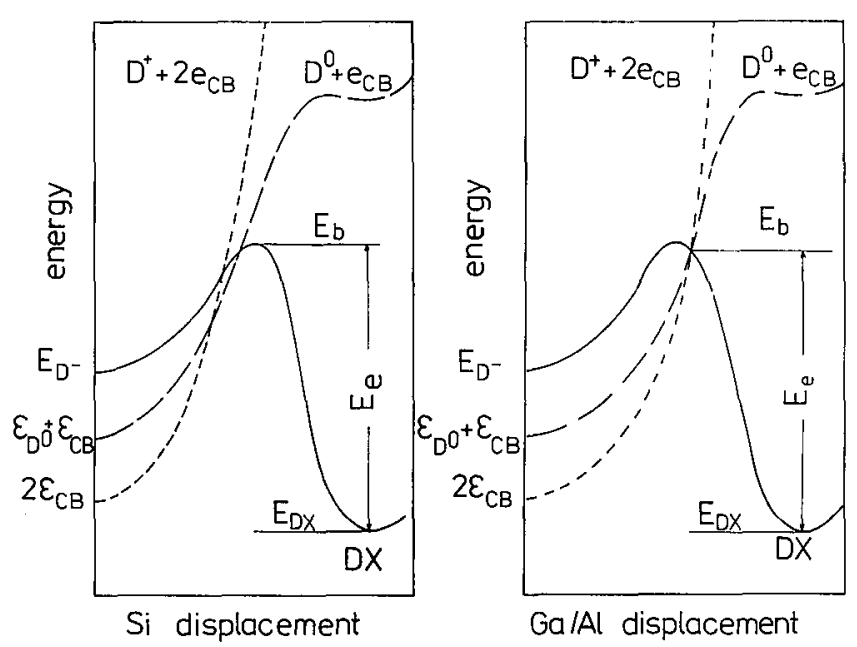

Fig. 1. The configurational diagram, i.e., the total energy of the one donor center and two electrons vs. lattice distortion is plotted. Solid line corresponds to the two-electron state; dashed line describes the sum of energies of the one-electron donor state $D^{0}$ and of energy of the conduction electron; dotted line corresponds to the sum of energies of two conduction electrons and the lattice distortion around the ionized center, $D^{+}$. The diagram 1a corresponds to Si-like DX while diagram $1 \mathrm{~b}$ to a Te-DX.

$D^{0}+$ e $_{\text {CB }}$ state. This transition occurs without any change of the lattice distortion. The transition energy is expected to depend on the CB energy and the observed dependences of the photoionization edge on alloy composition [18] and applied hydrostatic pressure (HP) [19] confirm the proposed model.

An additional comment is needed for the $D^{0}$ state in an unrelaxed lattice. The energy of this state is higher than that of the shallow donor state. From this point of view it is an excited state of the one-electron configuration of the donor. This state is important when it becomes the ground state. It happens when strong IIP is applied and the shallow donor state is shifted to higher energies - following the $\Gamma$ conduction band - or for heavily doped samples above the Mott transition. Wasilewski, Stradling and Dmochowski [20,21] observed (in the photoluminescence under HP an one-electron state confirming the population of the $D^{0}$ state as soon as its energy level is pushed below the conduction band minimum and the shallow donor level. Very recent, not yet published [22], experimental data confirm also the existence (weak hybridization) of this well-localized $D^{0}$ state when it is degenerate with CB. For GaAs:Ge $D^{0}$ is located below the DX state and when IIP is applied, a population of $D^{0}$ can be seen.

The main difference between Figs. $1 \mathrm{a}$ and $1 \mathrm{~b}$ is the type of the barrier which leads to metastability effects (the fingerprint of the DX-centers). Below we present the experimental data, mainly connected with emission and capture kinetics, which show that CD depicted in Fig. 1a corresponds to the Si-like DX-center while that in Fig. $1 \mathrm{~b}$ to DX with a group-VI donor. 


\section{Emission energy}

In Fig. 1a the top of the barrier corresponds to the maximum energy of the two-electron state. In the first step of thermal ionization of the DX-state the system has to be excited from the ground state to an excited state of the two-electron system. This step needs an activation energy $E_{\mathrm{e}}$ equal to the difference between the top of the energy barrier $E_{\mathrm{b}}$ and the ground state $E_{\mathrm{Dx}}{ }^{*}$. In the next step the two electrons are emitted, but this process does not need any energy. In consequence, the emission rate corresponding to the diagram 1a should be described simply by $c_{\mathrm{e}}=c_{\mathrm{e}}^{0} \exp \left(-E_{\mathrm{e}} / k_{\mathrm{B}} T\right)$, where the pre-exponential factor, $c_{\mathrm{e}}^{0}$, is temperature independent. At least, it should not contain any factor which comes from the temperature dependent density of CB states, since there is no activation to the CB states in the first step. This is the reason why the usually reported emission energies, which are evaluated with the use of a standard procedure (where a $T^{2}$ pre-factor is assumed), differ from that marked in the diagram by the energy $2 k_{\mathrm{B}} T$. After a necessary correction of the experimental data one can produce a "universal" Arrhenius plot [23]. In Fig. 2 the emission rate for AlGaAs:Si versus

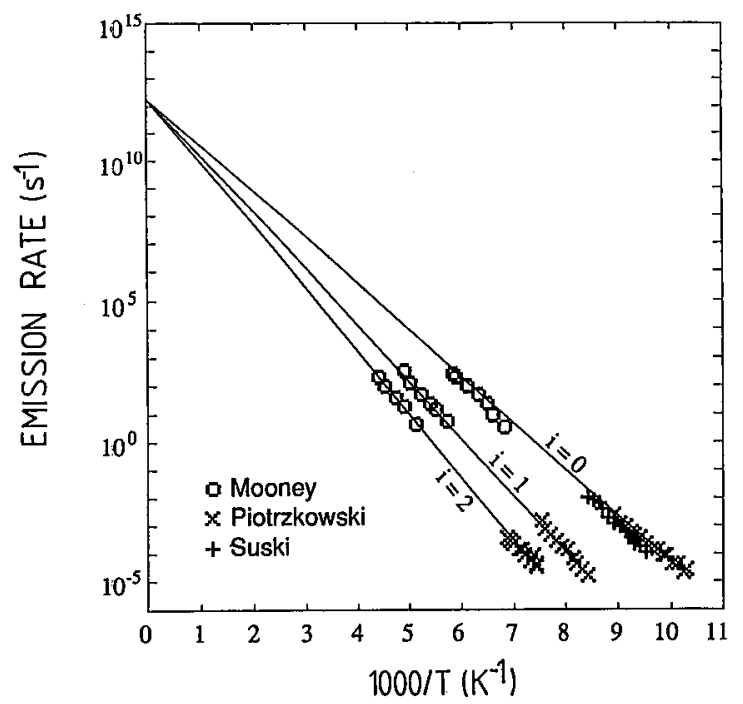

Fig. 2. Arrhenius plot of the emission rate of Si-DX centers in AlGaAs as obtained from DLTS [25] and high pressure transport kinetics [26].

inverse temperature is plotted. The alloy splitting of the emission energy due to various cation environments ( $\mathrm{Ga}$ or $\mathrm{Al}$ ) of the displaced $\mathrm{Si}$ atom is well seen (see Sec. 5). The deep level transient spectroscopy (DLTS) data measured with the constant capacity technique by Mooney et al. [24] and the electron emission rate

*The capital letter $E$ is used to describe a two-electron energy while the script $\mathcal{E}$ represents one-electron energy. Thus $E_{\mathrm{DX}}=2 \mathcal{E}_{\mathrm{DX}}, E_{\mathrm{b}}=2 \mathcal{E}_{\mathrm{b}}$, and so on. 
measured by Piotrzkowski et al. [25] during a warming process, after pressure induced filling of the DX-states, can be described by common parameters $c_{\mathrm{e}}^{0}$ and $E_{\mathrm{e}}$. The latest data of Dobaczewski et al. [26], where a Laplace analysis of the DLTS transients [27] has been done, are not presented in this figure. All these results show a well-resolved alloy splitting effect, however, the latter measurements were performed with constant voltage technique which could lead to some ambiguity. These results are shifted from the other data and we cannot easily re-evaluate them.

The thermal emission process corresponding to the diagram $1 \mathrm{~b}$ is much more complicated. Here, the top of the barrier constitutes the crossing of the two-electron state $\left(D^{-}\right)$with the states partially $\left(D^{0}\right)$ or fully $\left(D^{+}\right)$ionized. In the first step of thermal emission, which is activated by an emission energy, not only the excitation of local lattice vibration but also an ionization process takes place. In consequence, the emission rate should be described in much more complicated way. The emission energy is expected to depend on the CB energy. Since the latter depends on alloy composition $x$, pressure $p$, and temperature $T$, also the emission energy should depend on these parameters. The type of barrier involved can be tested experimentally by analyzing the influence of the $\mathcal{E}_{\mathrm{CB}}$ energy on the emission energy. It is well known that the emission energy for Si-like DX-centers in AlGaAs depends neither on the alloy composition nor on hydrostatic pressure $[1,28]$. Since both composition and pressure change the energy gap, and hence the $\mathcal{E}_{\mathrm{DX}}-\mathcal{E}_{\mathrm{CB}}$, one can conclude that Si-like DX-centers should be described by the barrier shown in the diagram 1a. Indeed, in this case $\mathcal{E}_{\mathrm{CB}}$ does not affect the energy of the top of the barrier $E_{\mathrm{b}}$ and a constant emission energy is expected.

DX-centers with donor of the group-VI donors exhibit other properties suggesting that for them the type of barrier shown in Fig. 1b is valid. Thus, the dependence of the emission rate on pressure and alloy composition as well as temperature dependence of both the pre-exponential factor and the emission energy (e.g. because of the temperature dependence of $\mathcal{E}_{\mathrm{DX}}-\mathcal{E}_{\mathrm{CB}}$ ) could be explained. These properties indicate that the experimental data should be very carefully analyzed when the emission rate from DX-centers induced by group-VI elements is investigated. It might explain the inconsistency obtained by us when we tried to construct "universal" Arrhenius plot (similar to that in Fig. 2) using the available experimental data for tellurium doped AlGaAs [26, 29].

\section{Capture process}

From the very beginning it was known that capture transients are very nonexponential implying a complexity of the capture process [30-32]. It is reflected in the configurational diagram, which shows the following features.

(i) There are many different paths of electron capture (see Fig. 1 where several possible sequences of the electron capture and lattice excitation results in the same total activation energy).

(ii) The energy needed for electron capture depends on the initial energy of the two electrons and is given by the difference between the top of the barrier $E_{\mathrm{b}}=2 \mathcal{E}_{\mathrm{b}}$ and two Fermi energies $2 \mathcal{E}_{\mathrm{F}}$. This is the main origin of the 
transient nonexponentiality since the quasi-Fermi energy changes during the transients. On the other hand, because of this fact it was possible to prove experimentally that two electrons are indeed captured on the DX-center. Jantsch et al. [23, 32] showed that the barrier height per one electron is two times smaller than the barrier per capture or emission event. Mosser et al. [33] measured directly the capture efficiency showing that the capture energy varies with two Fermi energies during the capture transients. Both experiments were done for Si doped AlGaAs. Diagram 1b suggests that the capture process for Te-like DX-centers is even more complicated, especially when the Fermi level is degenerate with the conduction band.

(iii) Another source of the capture nonexponentiality are the Coulomb potential fluctuations originating from the impurity charges [30-32]. This potential acts on the localized electron states and adds to its energies, $\mathcal{E}_{\mathrm{DX}}, \mathcal{E}_{D^{0}}, \mathcal{E}_{b}$, while the Fermi energy, $\mathcal{E}_{\mathrm{F}}$, is uniform in space. In consequence, potential fluctuations affect also the capture energy, which is given by the difference $E_{\text {cap }}=2\left(\mathcal{E}_{b}-\mathcal{E}_{\mathrm{F}}\right)^{*}$. Thus, the capture barrier varies in space while its mean value depends on the filling of DX-states by electrons [30,31].

(iv) The alloy splitting of the energy levels (see Sec. 5) leads to a new class of kinetics where capture and emission processes occur simultaneously (see Sec. 6).

\section{Alloy splitting}

The effect of alloy splitting can be discussed from two diametrally opposed points of view. Firstly, its occurrence supplied the crucial arguments proving the correctness of the model of Chadi and Chang [14]. Recent papers report on four and seven components of the emission processes in AlGaAs samples doped with Si [24-28] and Te [26, 29] respectively. Secondly, the variety of DX levels and activation energies resulting from alloy splitting increases the difficulties in treating of the experimental data.

Formation of the Si-related DX-center is connected with the large lattice displacement of the donor. It moves from the substitutional site as far along one of its bonds and there are three cations in its immediate vicinity. Depending on the number of $\mathrm{Ga} / \mathrm{Al}$ cations, four possible configurations can occur, which is assumed to be responsible for the fourfold split emission energy observed experimentally for $\mathrm{Si}$-induced DX-center. For Te-DX center it appears that one of the cations closest to $\mathrm{Te}(\mathrm{Ga}$ or $\mathrm{Al})$ is forced to move in the manner described above, leading to a twofold alloy splitting. Since there are four possible configurations for the three cations in the immediate vicinity of the Te-donor, the total number of possible configurations is $2 \times 4=8$. The experimentally observed alloy splitting of the emission energy confirms the microscopic model of the DX-center and underlines the difference between the DX induced by group-IV and group-VI elements.

The alloy splitting of the emission energy $E_{\text {em }}$ determines neither the splitting of the top of the barrier $E_{\mathrm{b}}$ (and, in consequence, the capture energy) nor

\footnotetext{
${ }^{*}$ See previous note.
} 
the splitting of the ground state of the DX-center, $E_{\mathrm{DX}}$, but only their difference. To find the total energy structure, additional data are required. The splitting of the top of the barrier could be visible in capture processes while the splitting of the ground state can be evaluated by measuring the number of electrons emitted from various sublevels at thermal equilibrium. Mooney et al. [24] and recently Su and Farmer [34] analyzed the amplitude of the DLTS peaks. In junction spectroscopy, however, it is very difficult to reach equilibrium during the filling pulse. Only the investigation of Su and Farmer [34] were performed for a wide range of filling pulses. Another set of experimental data was presented by Piotrzkowski et al. [25]. They analyzed the occupation change of the various sublevels under HP. Unfortunately, in this analysis the sublevel broadening was not considered, which leads to an error of the order of the potential fluctuation amplitude. It cannot be neglected in comparison to the alloy splitting being of the same order [30, 31].

Looking at all the available experimental data for Si-induced DX in AlGaAs, one can conclude that the energy of the top of the barrier is very similar for all types of Si-DX centers. The estimated splitting of this energy is a few $\mathrm{meV}$, i.e. less than the fluctuation of the Coulomb potential. It means that the splitting of the emission energy is determined by the splitting of the ground state energy. Unless contradicting experimental data are reported, the energy diagram presented by Wilamowski et al. [30,31] still does not need any correction.

For Te-like DX-centers there are no systematic experimental data concerning the ground state splitting. Some indications of a splitting of the top of the barrier can be found after a reinterpretation of the two stages of photoionization transients [35].

\section{Multi-stages kinetics}

According to the Chadi-Chang model [14], there are four equivalent directions of lattice distortion. This means that the DX-state and the states which constitute the top of barrier are fourfold degenerate for each type of DX-center in GaAs crystal. In alloys this degeneracy can be partially removed leading to four substates. The crucial point is that any transition between the substates of the same center, e.g. when thermalization is considered, can go only via $\mathrm{CB}$, by way of emission and recapture of both electrons. This property is reflected in the very specific "many stages" [31] kinetics. During the transient, because of the change of the quasi-Fermi level or temperature, the efficiencies of the various elementary capture and emission processes change dramatically leading to a different character of the transients at various stages. Their observation constitutes a new fingerprint of the DX model. On the other hand, it can lead to misunderstanding and misinterpretation of the experimental results.

In particular, the capture rate oscillations appearing during the warming of the previously illuminated AlGaAs:Si sample [36] is a well-evidenced multi-stage process $[30,31,37]$. The observed oscillations are not connected with the splitting of the capture barrier but with the quantization of the emission rate which dominates the process at some stages.

The capture kinetics for Te-induced DX have not been investigated. A few 
years ago, however, Dobaczewski and Kaczor. [35] systematically investigated the photoionization transients in Te doped AlGaAs. They observed very characteristic nonexponential transients with specific overshoots. It is clear that such dependence can be explained when more than one stage of the process is assumed. Originally, the authors interpreted the results as a "proo" of the negative $U$ character of the DX-center claiming that the two stages correspond to photoemission of the first and of the second electron. New data which show strong alloy splitting of the emission energy, done also by Dobaczewski et al. [26], indicate now a new possibility of interpretation of the old photoionization results [35]. A preliminary analysis shows that the characteristic family of transients can originate from alloy splitting of the top and of the ground state of the DX-center. If one assumes that the substate which is characterized by a smaller emission energy has a higher lying ground state and a lower top of the barrier, then it is clear that the photoemission process consists of two stages. In the first stage photoionization of the lower ground state takes place, the quasi-Fermi energy increases making recapture process possible. But the recapture on the state, characterized by a lower top of the barrier and a higher ground state, is more effective. Thus, the second stage corresponds to an approaching of a steady state related to an equilibrium between electron photoemission and recapture on the upper ground state.

It is difficult to make any conclusion when quantitative description has not been made. The suggestion, however, that the energy of the top of the barrier for DX-centers induced by group-VI elements strongly depends on the replaced cation ( $\mathrm{Al}$ or $\mathrm{Ga}$ ) seems to be interesting to consider.

\section{Conclusions}

Concluding, it seems that the Si-induced DX is well described by the CD shown in Fig. 1a, i.e. that Si-DX is the two-electron state characterized by large lattice relaxation. Thus, the microscopic model of Chadi and Chang [14] and the calculations of Dabrowski et al. [15] are well confirmed experimentally. For group-VI donors the nature of the DX-center is similar to that of Si-DX but the detailed picture is not so clear. We hope that an analysis of the new experimental data by means of the proposed diagram $1 \mathrm{~b}$ would help to clarify the picture, provided that all the specific features of the DX-centers are taken into account.

\section{Acknowledgments}

The authors wish to thank L. Dobaczewski, P. Kaczor, J. Kossut, J.M. Langer, G. Ostermayer, and R. Piotrzkowski for helpful discussions.

\section{References}

[1] For review of the DX properties see: P.M. Mooney, J. Appl. Phys. 67, R1 (1990); Physics of DX Centers in GaAs Alloys, Ed. J.C. Bourgoin, Sci-Tech Publications, Liechtenstein 1990;DX-Centers and Other Metastable Defects in Semiconductors, Proc. Int. Symp. Mauterndorf, Eds. W. Jantsch, R.A. Stradling, A. Hilger, Bristol 1991 (this book appeared also as a volume of Semicond. Sci. Technol. 6). 
[2] G.A. Baraff, E.O. Kane, M. Schlutter, Phys. Rev. Lett. 43, 956 (1979); Phys. Rev. B 21, 3562 (1980).

[3] G.D. Watkins, J.R. Troxel, Phys. Rev. Lelt. 44, 593 (1980).

[4] For review see: G.D. Watkins, Semicond. Sci. Technol. 6, B 111 (1991).

[5] S. Porowski, M. Konczykowski, J. Chroboczek, Phys. Stalus Solidi B 64, 291 (1974).

[6] L. Dmowski, M. Konczykowski, R. Piotrzkowski, S. Porowski, Phys. Stalus Solidi B 73, K131 (1976).

[7] M. Baj, L. Dmowski, M. Konczykowski, S. Porowski, Phys. Status Solidi A 33, 421 (1976).

[8] D.W. Lang, R.A. Logan, Phys. Rev. Lett. 39, 635 (1979); D.V. Lang, R.A. Logan, M. Jaros, Phys. Rev. B 19, 1015 (1979).

[9] U. Piekara, J.M. Langer, B. Krukowska-Fulde, Solid Slate Commun. 23, 583 (1977).

[10] J.M. Langer, U. Ogonowska, A. Iller, Inst. Phys. Conf. Ser. 43, 295 (1979); for review see also J.M. Langer, Radiat. Eff. Defects Solids 72, 55 (1983).

[11] J.E. Dmochowski, J.M. Langer, Z. Kalinski, W. Jantsch, Phys. Rev. Lelt. 56, 1735 (1986).

[12] J.M. Langer, Rev. Solid State Sci. 4, 279 (1990).

[13] Y. Toyozawa, Solid State Electron. 21, 1313 (1977).

[14] D.J. Chadi, K.J. Chang, Phys. Rev. Lelt. 61, 873 (1988), Phys. Rcv. B 39, 10063 (1989).

[15] J. Dabrowski, M. Scheffler, R. Strehlow, in: Proc. ICPS-20, Eds. E.M. Anastassakis, J.D. Joannopulos, Word Scientific, Singapore 1990, p. 489; J. Dabrowski, M. Scheffler, Mater. Sci. Forum 83-87, 735 (1991).

[16] P.M. Mooney, T.N. Theis, Wright, Appl. Phys. Lett. 53, 2546 (1988). See also Ref. [1].

[17] J.E. Dmochowski, L. Dobaczewski, J.M. Langer, W. Jantsch, Phys. Rev. 40, 9671 (1989).

[18] J.A. Wolk, M.B. Kruger, J.N. Neyman, W. Walukiewicz, R. Jeanioz, E.E. IIaller, Semicond. Sci. Technol. 6, B78 (1991).

[19] T. Suski, M. Baj, Phys. Scr. Vol. T 39, 250 (1991).

[20] Z. Wasilewski, R.A. Stradling, S. Porowski, Solid State Commun. 57, 123 (1986); J. Phys. E 19, 480 (1986).

[21] J.M. Dmochowski, R.A. Stradling, P.D. Wang, S.N. Holmes, M. Li, B.D. McCombe, B. Weinstein, Semicond. Sci. Technol. 6, 476 (1991).

[22] P. Van der Wel, P. Wisniewski, C. Skierbiszewski, T. Suski, to be published.

[23] W. Jantsch, Z. Wilamowski, G. Ostermayer, Semicond. Sci. Technol. 6, B47 (1991); Mater. Sci. Forum 83-87, 799 (1991).

[24] P.T. Mooney, T.N. Theis, S.L. Wright, Appl. Phys. Lelt. 53, 2546 (1988). See also Ref. [1].

[25] R. Piotrzkowski, E. Litwin-Staszewska, J.L. Robert, V. Mosscr, P. Lorenzini, Semicond. Sci. Technol. 6, 500 (1991); R. Piotrzkowski, E. Litwin-Staszewska, P. Lorcnzini, J.L. Robert, Semicond. Sci. Technol. 7, 103 (1992).

[26] L. Dobaczewski, P. Kaczor, M. Missous, A.R. Peaker, Z. Zylkiewicz, Phys. Rev. Lett., 1992, to be published. 
[27] L. Dobaczewski, I.D. Hawkins, P. Kaczor, M. Missous, I. Poole, A.R. Peaker, Maler. Sci. Forum 83-87, 769 (1991).

[28] R. Piotrzkowski, T. Suski, P. Wisniewski, K. Plog, J. Knecht, J. Appl. Phys. 68, 3377 (1990).

[29] R. Piotrzkowski, E. Litwin-Staszewska, T. Suski, L. Konczewicz, J.L Robert, W. Stankiewicz, submitted to Appl. Phys. Lett., 1992.

[30] Z. Wilamowski, J. Kossut, W. Jantsch, G. Ostermayer, Semicond. Sci. Technol. 6, B38 (1991).

[31] Z. Wilamowski, W. Jantsch, G. Ostermayer, J. Kossut, Mater. Sci. Forum 83-87, 805 (1991).

[32] W. Jantsch, Z. Wilamowski, G. Ostermayer, to be published in Phys. Scr., 1992, Proc. EPS Meeting, Prague 1992.

[33] V. Mosser, S. Contreras, J.L. Robert, R. Piotrzkowski, W. Zawadzki, J.F. Rochette, Phys. Rev. Lett. 66, 1737 (1991).

[34] Z. Su, J.W. Farmer, Mater. Sci. Forum 83-87, 817 (1991); Appl. Phys. Lelt. 59, 1746 (1991).

[35] L. Dobaczewski, P. Kaczor, Phys. Rev. Lell. 66, 68 (1991); Phys. Rev. B 44, 8621 (1991).

[36] G. Brunthaler, K. Kohler, Appl. Phys. Lett. 57, 2227 (1990).

[37] G. Brunthaler, G. Ostermayer, A. Falk, W. Jantsch, Z. Wilamowski, Mater. Sci. Forum 83-87, 823 (1991). 